\title{
Comparative evaluation of 15 -minute rapid diagnosis of ischemic heart disease by high-sensitivity quantification of cardiac biomarkers
}

\author{
SHENGNAN HE* , LONG QU* ${ }^{*}$ XI HE, DAN ZHANG and NI XIE \\ Institute of Translational Medicine, Health Science Center, Shenzhen Second People's Hospital, The \\ First Affiliated Hospital of Shenzhen University, Shenzhen, Guangdong 518035, P.R. China
}

Received September 4, 2019; Accepted April 17, 2020

DOI: $10.3892 /$ etm.2020.8853

\begin{abstract}
A rapid clinical diagnosis may efficiently improve the survival rate and prognosis of patients with ischemic heart disease (IHD). Therefore, a one-step, rapid and inexpensive analysis for the quick diagnosis of IHD was investigated in the present study. Consecutive patients who were subjected to myoglobin, cardiac troponin I and creatine kinase-MB isoenzyme assessment at the Emergency Department of Shenzhen Second People's Hospital (Shenzhen, China) between December 2017 and March 2018 prior to treatment were screened. Clinically applicable disposable strips were employed for quantification of the cardiac biomarkers. The analytical performance of the strips was evaluated by receiver operating characteristic (ROC) curves and compared with the traditional chemiluminescence immunoassay (CLIA) method. The data of 391 participants were collected. At the baseline, 57 patients were diagnosed with IHD and 334 patients were diagnosed with other diseases. The area under the ROC curve (AUC) of the CLIA model was 0.787 (95\% CI, 0.709-0.865) with a specificity of $76.7 \%$ and a sensitivity of $71.9 \%$. At the optimal cutoff value of -1.867 , the negative and the positive predictive value were 94.1 and $34.5 \%$, respectively. The AUC of the disposable
\end{abstract}

Correspondence to: Professor Ni Xie, Institute of Translational Medicine, Health Science Center, Shenzhen Second People's Hospital, The First Affiliated Hospital of Shenzhen University, 3002 Sungang West Road, Shenzhen, Guangdong 518035, P.R. China E-mail: xn100@szu.edu.cn

*Contributed equally

Abbreviations: AUC, area under the ROC curve; CLIA, chemiluminescence immunoassay; CK-MB, creatine kinase-MB isoenzyme; cTnI, cardiac troponin I; IHD, ischemic heart disease; Myo, myoglobin; POCT, point-of-care testing; RIA, radioimmunoassay; ROC, receiver operating characteristic

Key words: ischemic heart disease, cardiac biomarkers, rapid diagnosis, disposable strips strip model was 0.792 (95\% CI, 0.729-0.855). At the cutoff value of -1.820 or below, the negative predictive value was $94.9 \%$, the positive predictive value was $28.9 \%$, the specificity was $66.8 \%$ and the sensitivity was $79.0 \%$. The P-value of the ROCs was 0.858 , indicating no statistically significant difference between the two assay methods. The cost of the disposable strip was $50 \%$ of that of the CLIA method and it took only $25 \%$ of the time that was required for the quantification of the three cardiac markers by CLIA. In conclusion, the disposable strip provides a platform for point-of-care testing and may be an easy, rapid, reliable and cost-saving method for the diagnosis of IHD.

\section{Introduction}

Ischemic heart disease (IHD) is an important global public health concern, which is the most common cause of mortality and disability worldwide (1-3). An estimated 110.55 million prevalent cases of IHD were present globally in 2015 (4). The World Health Organization estimates further increases in IHD-associated mortality by 2030 in all country income strata (5). Therefore, IHD remains a huge burden on healthcare systems.

Early diagnosis may efficiently improve the survival rate and prognosis of patients with IHD, which relies on medical history, electrocardiogram and cardiac biomarkers (6). As clinical history and physical examination of patients are limited and 12-lead electrocardiogram is only used for diagnosis in a small proportion of cases, the use of specific biomarkers as diagnostic indicators has added value for rapidly diagnosis of IHD in populations (7-11). Myoglobin (Myo), cardiac troponin I (cTnI) and creatine kinase-MB isoenzyme (CK-MB) are common biomarkers frequently used for IHD diagnosis in hospital settings $(8,12-14)$. The concentration of these cardiac biomarkers may be determined by radioimmunoassay, ELISA and chemiluminescence immunoassay (CLIA) with excellent specificity and sensitivity $(15,16)$. At present, CLIA is commonly used in hospitals. However, the complex procedures take a large amount of time and rely on bulky instruments, which may delay the rescue of patients and are inconvenient to perform. Therefore, the rapid detection of these biomarkers with a portable device may be an efficient approach for better prognostic prediction. 
Disposable strips based on a fluorescent immunoassay are generally used for the quantitative determination of specific markers (17). This method allows for a one-step, rapid and inexpensive analysis, which is easy to perform at community health centers and in under-developed regions. In the present study, the strips for the quantification of Myo, cTnI and $\mathrm{CK}-\mathrm{MB}$ were employed for a substantial reduction in the time of IHD diagnosis.

\section{Materials and methods}

Materials. Myo, cTnI and CK-MB disposable strips and a UNICELL immunofluorescence reader were obtained from Shenzhen YHLO Biotech.

UNICELL-cTnI/CK-MB/Myo employs an immuno-chromatography sandwich assay that generates a fluorescence signal, which is proportional to the level of cTnI/CK-MB/Myo in the blood specimen. Corresponding mouse anti-cTnI/CK-MB/Myo antibody is coated on the test line ( $\mathrm{T}$ line) of the testing strip and anti-avidin antibody is coated on the control line $(\mathrm{C}$ line) of the testing strip. Fluorescence particles labeled anti-cTnI/CK-MB/Myo antibody and avidin are coated on the binding pad (Shenzhen YHLO Biotech Co. Ltd.).

Whole blood samples were obtained from patients at Shenzhen Second People's Hospital (Shenzhen, China). Testing was performed by adding $100 \mu \mathrm{l}$ whole blood, serum or plasma into the sample well. If cTnI/CK-MB/Myo was present in the specimen, an immune complex is formed by binding cTnI/CK-MB/Myo with fluorescence particle-labelled anti-cTnI/CK-MB/Myo antibody. Subsequently, through the capillary effect, this immune complex moves forward to the $\mathrm{T}$ line and is captured by another anti-cTnI/CK-MB/Myo antibody on it. At the same time, the fluorescence particle-labeled avidin on the binding pad passes through the $\mathrm{C}$ line and binds with the anti-avidin antibody coated on the $\mathrm{C}$ line.

The UNICELL immunofluorescence reader was used to determine the signal intensity of fluorescence of the $\mathrm{T}$ line and the $\mathrm{C}$ line. The concentration of $\mathrm{cTnI} / \mathrm{CK}-\mathrm{MB} / \mathrm{Myo}$ in the specimen was automatically calculated according to the master curve that was stored in the ID card and the result was displayed in the units of $\mathrm{ng} / \mathrm{ml}$.

Patient recruitment and data collection. For the present clinical validation study, consecutive patients who had been subjected to assessment of Myo, cTnI and CK-MB by CLIA at the Emergency Department of Shenzhen Second People's Hospital (Shenzhen, China) between December 2017 and March 2018 were subjected to screening with the test strips. Patients were excluded if they had received medical treatment prior to blood collection or were without complete data. A total of 391 randomized patients were enrolled in the study, ranging from 15 to 96 years. A total of $62.40 \%$ of the patients were male. Baseline data were collected by trained research staff according to a standard operating procedure. The data included gender, age, the concentrations of Myo, cTnI and CK-MB quantified by CLIA and disposable strips. The study was performed in accordance with the Declaration of Helsinki and was approved by the Ethics Committee of Shenzhen Second People's Hospital
Table I. Baseline characteristics of the patients $(n=391)$.

\begin{tabular}{lc}
\hline Clinical characteristic & Value \\
\hline Age (years) & $62.9(49.5-77)$ \\
Male sex & $244(62.40)$ \\
Diagnosed diseases & \\
Ischemic heart disease & $57(14.58)$ \\
$\quad$ Acute myocardial infarction & $23(5.88)$ \\
Acute coronary syndrome & $21(5.37)$ \\
Acute heart failure & $4(1.02)$ \\
Acute myopericarditis & $1(0.26)$ \\
Cardiogenic shock & $1(0.26)$ \\
Cardiopulmonary arrest & $1(0.26)$ \\
Other diseases & $334(85.42)$ \\
\hline
\end{tabular}

Values are expressed as $\mathrm{n}(\%)$ or median (25th-75th percentile).

(Shenzhen, China; approval no. 20170811015). During data collection, all patients were informed to fully understand the study and signed the consent documents referenced from the Chinese Clinical Trial registry (http://www.chictr.org. $\mathrm{cn} /$, no. ChiCTR2000032549). All of the experiments were performed in accordance with relevant guidelines and regulations.

Quantification methods. The concentrations of $\mathrm{Myo} / \mathrm{cTnI} / \mathrm{CK}-\mathrm{MB}$ in the blood samples were determined with the disposable strips and quantitatively evaluated with the UNICELL immunofluorescence reader. The procedure was as follows: All of the blood samples were stored at $4^{\circ} \mathrm{C}$ for $24 \mathrm{~h}$. The samples were left to reach room temperature for $10 \mathrm{~min}$ prior to further testing. Each sample $(100 \mu \mathrm{l})$ was dispensed into the sample well and the strips were then inserted into the reader. The fluorescent signal intensities of the $\mathrm{T}$ line and the $\mathrm{C}$ line were recorded $15 \mathrm{~min}$ later, which were used to calculate the concentrations of Myo/cTnI/CK-MB in the samples according to the master curve that is stored in the ID Card. The values $(\mathrm{ng} / \mathrm{ml})$ were displayed automatically on the screen of the device. The Myo/cTnI/CK-MB quantification results by the CLIA method were obtained from the department of laboratory medicine of Shenzhen Second People's Hospital (Shenzhen, China).

Statistical analysis. In the setting of the present study, a total of 57 patients diagnosed with IHD would provide a statistical power of $99 \%$ to validate the use of test strips for cardiac biomarkers. Baseline characteristics, including gender, age and the concentrations of Myo, cTnI and CK-MB quantified by CLIA and disposable strips, were analysed to construct the receiver operating characteristic (ROC) curves, which were used to determine the optimal cut-off concentrations and compare the sensitivity and specificity of different cardiac biomarkers and their predictive value to diagnose IHD. The ROC curves with or without bootstrap resampling (time $=500)$ and a nomogram were established with EmpowerStats software (www.empowerstats. com, X\&Y solutions, Inc.; updated on 28/02/2020). 


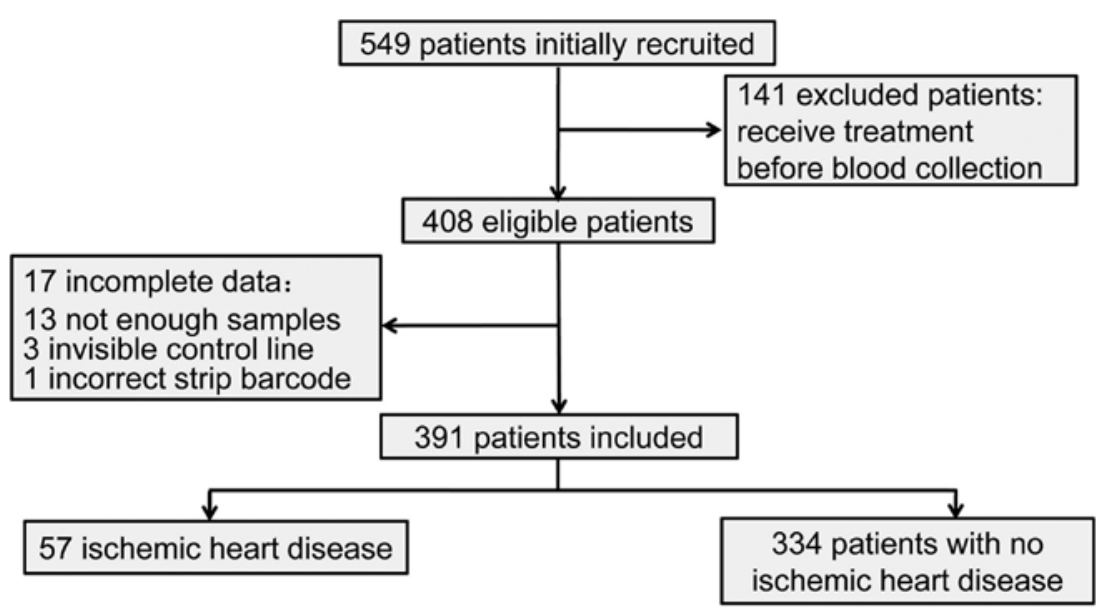

Figure 1. Flow chart of participant recruitment. There were 549 patients initially recruited, and 141 patients were excluded because they received treatment before blood collection. There were 17 participants excluded due to an insufficient blood volume for completing the test, invisible control line or incorrect strip barcode that cannot be recognized by the immunofluorescence reader. The final study population comprised 391 patients, and 57 patients were diagnosed with IHD at the baseline. IHD, ischemic heart disease.

\section{Results}

Patients. A total of 549 participants from the Emergency Department received Myo/cTnI/CK-MB tests during the study period and 141 of them were excluded due to having received treatment prior to blood collection. Another 17 participants were excluded due to an insufficient blood volume for completing the test, invisible control line or incorrect barcode. The final study population comprised 391 patients. The median age was $62.9 \pm 17.21$ years and $62.40 \%$ of the patients were male (Table I). At baseline, 57 patients were diagnosed with IHD, including 23 cases of acute myocardial infarction, 21 of acute coronary syndrome, 4 cases of acute heart failure, 1 of acute myopericarditis, 1 of cardiogenic shock and 1 case of cardiopulmonary arrest. The remaining 334 patients were diagnosed with other diseases (Fig. 1; Table I).

Comparison of the CLIA model and disposable strip model. A total of 2 different multivariable logistic regression models were constructed. Model 1 was constructed with baseline predictors, including gender, age and concentration of Myo/cTnI/CK-MB assessed by CLIA method. Model 2 included gender, age and concentration of Myo/cTnI/CK-MB quantified by disposable strip. The regression coefficients from the multivariate logistic model were used to construct the model for predicting the presence of IHD. The scoring model 1 was as follows: $-1.22037+0.17693 \mathrm{x}$ gender $-0.01179 \mathrm{x}$ age $+0.46488 \mathrm{x}$ cTnI $-0.00207 \times \mathrm{xyo}+0.00102 \times$ CK-MB. The scoring model 2 was as follows: $-0.95456+0.16130 \mathrm{x}$ gender $-0.00966 \mathrm{x}$ age $+0.19297 \mathrm{x}$ cTnI $-0.01119 \mathrm{x}$ Myo $+0.02895 \mathrm{x}$ CK-MB (sex: ' 0 ' for male and ' 1 ' for female; 'years' for age; 'ng/ml' for the laboratory parameters). ROC analysis was used to identify the area under the ROC curve (AUC) of each model (Fig. 2A). The AUC of model 1 was 0.787 (95\% CI, $0.709-0.865$ ) with a specificity of $76.7 \%$ and a sensitivity of $71.9 \%$ at a cutoff value of -1.867 . The AUC of model 2 was 0.792 (95\% CI, 0.728-0.855). The specificity was $66.8 \%$ and the sensitivity was $79.0 \%$. The optimal cutoff value was determined to be -1.820 . There was no significant difference between model 1 and model 2 in terms of IHD diagnosis $(\mathrm{P}=0.858)$. Parameters regarding the predictive value of the disposable strip test for IHD are presented in Table II. The negative predictive value at the cutoff value of -1.820 or below for the scoring model 2 was $94.9 \%$ and the positive predictive value was $28.9 \%$. The results indicated that with a cutoff value of -7.008 , the sensitivity and negative predictive value for the diagnosis of IHD were 98.3 and $98.8 \%$, respectively.

ROC curves for the diagnosis of IHD in each model using Bootstrap resampling (times $=500$ ) indicated that the AUC of model 1 was 0.78 (95\% CI, 0.6899-0.8519; specificity, 76,65\%; sensitivity, 71,93\%). The AUC of model 2 was slightly higher than that of model 1 (AUC $=0.79 ; 95 \%$ CI, 0.6899-0.8519; specificity, $66.77 \%$; sensitivity, $78.95 \%$ ). The $\mathrm{P}$-value was $0.7465(\mathrm{P}>0.05)$, indicating that the disposable strip model shared comparable accuracy in IHD diagnosis with the CLIA model (Fig. 2B-D). ROC curves for the individual biomarkers quantified by disposable strip are provided in Fig. 3. Compared with the combined use of the biomarkers (AUC $=0.792$ ), the AUC for single use of Myo, cTnI and CK-MB was $0.685(\mathrm{P}<0.001), 0.706(\mathrm{P}=0.0092)$ and 0.662 $(\mathrm{P}<0.001)$, respectively. The result suggested that the predictive performance of Myo, cTnI and CK-MB, used separately, was not superior to the predictive performance of the combination use. To predict the presence of IHD, a prognostic nomogram was established through Cox regression model analysis according to all significant independent indicators, including gender, age and the concentration of Myo, cTnI and CK-MB quantified by the disposable strip. Each factor in the nomogram was assigned a weighted number of points and the sum of points for each patient reflected the probability of the presence of IHD (Fig. 4).

The disposable strip is a cost-saving and rapid method for IHD diagnosis. The cost of the two methods was compared. The patients paid 47.18 US\$ for the quantification of Myo/cTnI/CK-MB by CLIA, while the disposable 
Table II. Proportions of patients with positive samples, sensitivities, specificities, positive and negative predictive values and likelihood ratios for the disposable strip to identify patients with ischemic heart disease.

\begin{tabular}{lcccccc}
\hline Cut-off value & Specificity $(\%)$ & Sensitivity $(\%)$ & PPV $(\%)$ & NPV $(\%)$ & + likelihood ratio & - likelihood ratio \\
\hline-7.008 & 23.7 & 98.3 & 18.0 & 98.8 & 1.293 & 0.072 \\
-2.119 & 48.2 & 89.5 & 22.8 & 96.4 & 1.733 & 0.217 \\
-1.820 & 66.8 & 79.0 & 28.9 & 94.9 & 2.382 & 0.324 \\
-1.641 & 74.3 & 68.4 & 31.2 & 93.2 & 2.658 & 0.432 \\
-1.601 & 78.4 & 59.7 & 32.1 & 92.9 & 2.766 & 0.514 \\
\hline
\end{tabular}

The scoring model was as follows: $-0.95456+0.16130$ x gender -0.00966 x age +0.19297 x cTnI -0.01119 x Myo + 0.02895 x CK-MB (sex: ' 0 ' for male and ' 1 ' for female; 'years' for age; 'ng/ml' for the laboratory parameters). PPV, positive predictive value; NPV, negative predictive value; CK-MB, creatine kinase-MB isoenzyme; cTnI, cardiac troponin I; Myo, myoglobin.
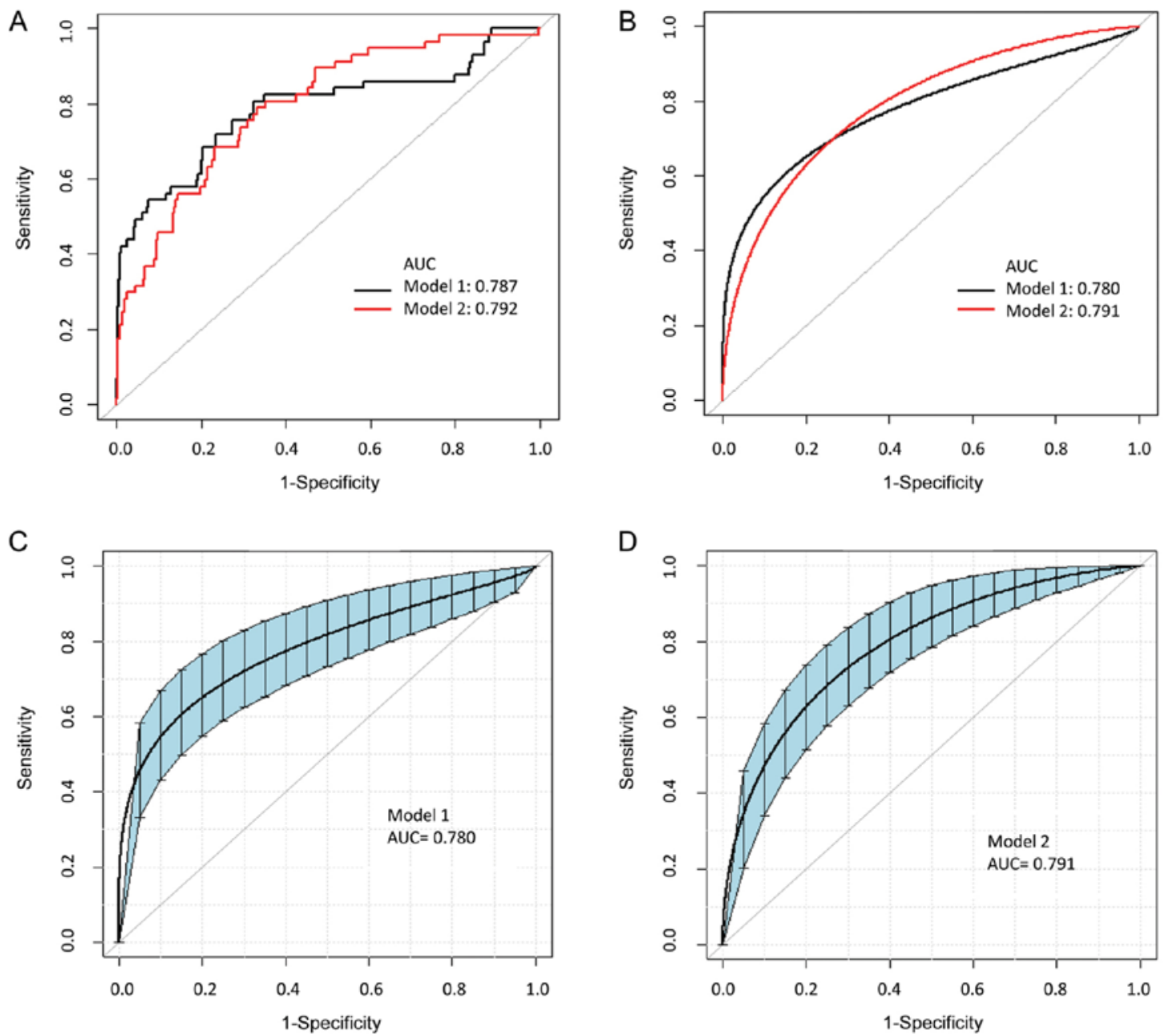

Figure 2. ROC curve for diagnosis of IHD. (A) Model 1 (black) is for the CLIA model including gender, age, concentration of Myo/cTnI/CK-MB assessed by the CLIA method. Model 2 (red) is for the disposable strip model including gender, age and concentration of Myo/cTnI/CK-MB quantified by disposable strips. The AUCs for model 1 and model 2 for predicting IHD were 0.787 and 0.792 . There was no significant difference between the two models. The optimal cutoff values of modle 1 and modle 2 were -1.867 and -1.820 . (B) ROC curves for the diagnosis of IHD in each model using Bootstrap resampling (times=500). The AUCs for model 1 (black) and model 2 (red) for predicting IHD were 0.780 and 0.791. (C) ROC curves for the diagnosis of IHD in model 1 using Bootstrap resampling (times=500). The optimal cutoff value was -1.867 . The blue area represented $95 \% \mathrm{CI}(0.710-0.854)$ of the AUC. (D) ROC curves for the diagnosis of IHD in model 2 using Bootstrap resampling (times=500). The optimal cutoff value was -1.820 . The blue area represented $95 \% \mathrm{CI}(0.729-0.845)$ of the AUC. ROC, receiver operating characteristic; AUC, area under the ROC curve; CLIA, chemiluminescence immunoassay; CK-MB, creatine kinase-MB isoenzyme; cTnI, cardiac troponin I; IHD, ischemic heart disease; Myo, myoglobin.

strip method only cost 21.91 US\$, saving $>50 \%$ compared to the CLIA method. The time spent on each method was also compared in the present study. The doctors of the emergency department usually waited for $>1 \mathrm{~h}$ to get the results of the 
A

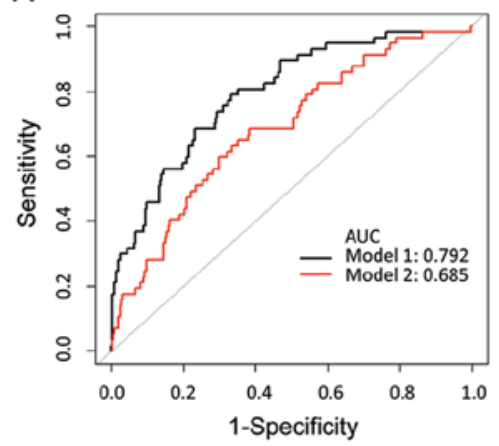

B

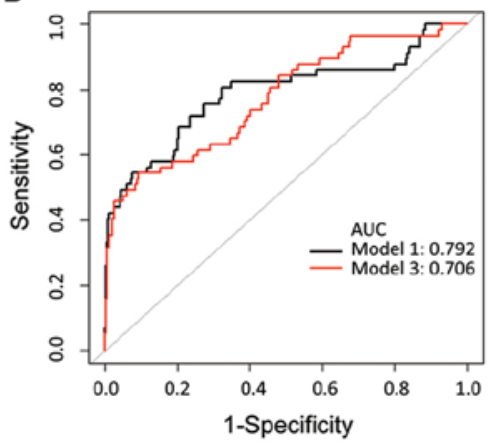

C

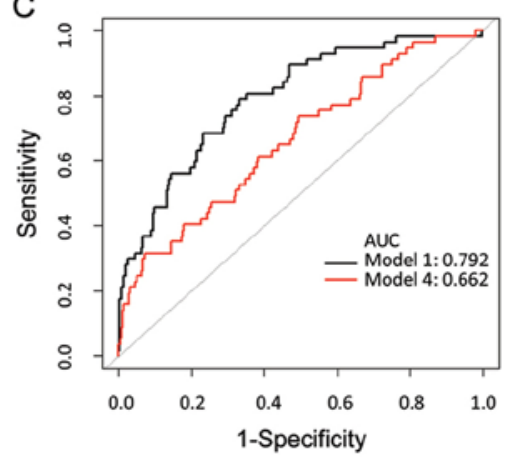

Figure 3. Comparison of the biomarkers applied individually and collectively. Model 1 (black) is the combination model including gender, age and concentration of Myo/cTnI/CK-MB quantified by disposable strip. Model 2, model 3 and model 4 (red) are single models including gender, age and concentration of (A) Myo, (B) cTnI or (C) CK-MB quantified by a disposable strip, separately. ROC, receiver operating characteristic; AUC, area under the ROC curve; CK-MB, creatine kinase-MB isoenzyme; cTnI, cardiac troponin I; Myo, myoglobin.

Points

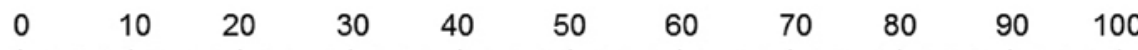

Gender $\quad \stackrel{\text { Female }}{\text { Male }}^{\text {Fem }}$

Age

100704010

cTnl, ng/ml

\begin{tabular}{lllllllll}
\hline 0 & 5 & 10 & 15 & 20 & 25 & 30 & 35 & 40
\end{tabular}

Myo, ng/ml

$\begin{array}{lllllllllll}500 & 450 & 400 & 350 & 300 & 250 & 200 & 150 & 100 & 50 & 0\end{array}$

$\mathrm{CK}-\mathrm{MB}, \mathrm{ng} / \mathrm{ml}$

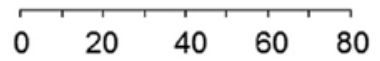

Total points

$\begin{array}{lllllllllllll}0 & 20 & 40 & 60 & 80 & 100 & 120 & 140 & 160 & 180 & 200 & 220 & 240\end{array}$

Probability of IHD presence

$0.10 .30 .50 .7 \quad 0.9$

Figure 4. Nomogram for estimating the risk of IHD presence in the cohort by disposable strip method. To use the nomogram, draw an upward vertical line from each variable axis to the 'Points' bar to determine the points of each variable. The points from all of the variables were added and a downward vertical line from the 'Total points' axis was drawn to determine the IHD probabilities at the lower line of the nomogram. The cardiac parameters were quantified by the disposable strip. CK-MB, creatine kinase-MB isoenzyme; cTnI, cardiac troponin I; IHD, ischemic heart disease; Myo, myoglobin.

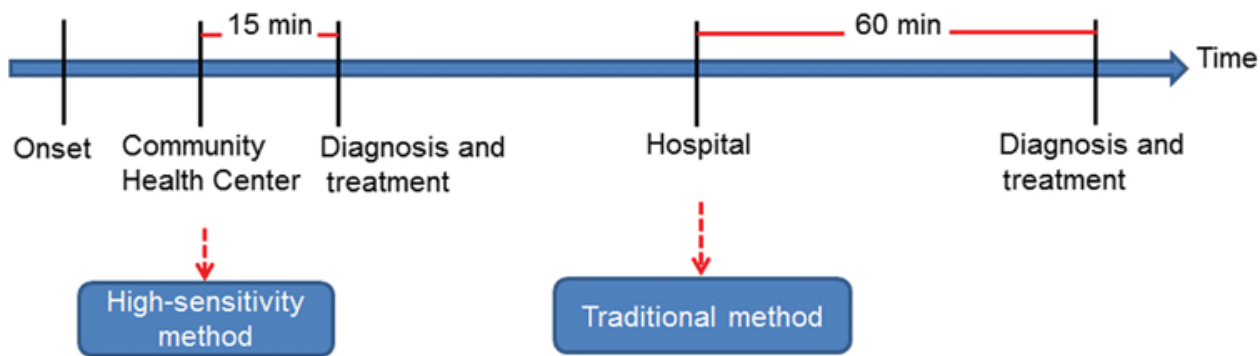

Figure 5. Scheme of the CLIA and disposable strip methods for the diagnosis of ischemic heart disease. The high-sensitivity method is quantification of Myo/cTnI/CK-MB by disposable strips; the traditional method is quantification of Myo/cTnI/CK-MB by CLIA. CLIA, chemiluminescence immunoassay; CK-MB, creatine kinase-MB isoenzyme; cTnI, cardiac troponin I; Myo, myoglobin. 
Myo/cTnI/CK-MB tests by CLIA, while it only took $15 \mathrm{~min}$ for the disposable strip method, which saved $75 \%$ of the time to diagnose and treat the patients with IHD earlier (Fig. 5).

\section{Discussion}

Disposable strips based on a fluorescent immunoassay have received considerable attention due to their portability, rapid performance, cost effectiveness and ease of use $(16,17)$. This qualitative assay is easy to perform and evaluate based on the fluorescence intensity of the band on the test zone, and the quantitative data may be obtained by recording the optical responses with a hand-held 'strip reader'. It has been derived to recognize a diverse range of targets, including metal ions (18), protein (19), cells (20) and toxicants (21), with high specificity and sensitivity. In the present study, a method to rapidly diagnose IHD was established using patient gender, age and the concentration of Myo/cTnI/CK-MB assessed by disposable strips. It exhibited a similar diagnostic capability for IHD compared with the traditional CLIA method frequently used in hospitals. Furthermore, the method of the present study had the following advantages: First, since cardiac myocytes may withstand ischemia for only $30 \mathrm{~min}$ prior to the occurrence of permanent injury, reliable and rapid tests for myocardial damage urgently require inclusion for rapid treatment. According to clinical guidelines, the test information of cardiac biomarkers is required within $1 \mathrm{~h}$ to diagnose patients and manage their treatment (22). Furthermore, the National Academy of Clinical Biochemistry Laboratory Medicine Practice guidelines have also suggested that laboratory testing for cardiac markers should be performed with a turnaround time of $1 \mathrm{~h}$, optimally $30 \mathrm{~min}$ or less $(23,24)$. In institutions unable to meet the 1-h turnaround time requirement, quantitative point-of-care testing (POCT) should be implemented $(23,24)$. As the conventional approach for IHD diagnosis takes $>1 \mathrm{~h}(25)$, the 15-min rapid method provided by the present study is time-saving, which greatly facilitates faster decision-making and interventional therapy. This assay is likely to improve the survival rate and prognosis of patients with IHD. Furthermore, the CLIA method requires bulky instruments, which is in conflict with the bedside-testing purpose of portable devices, while the system of the present study perfectly meets the requirement for POCT and demonstrated a reliable analytical performance. To this end, the disposable strip-based method of the present study may be widely applied in under-developed regions and community health service centers and at marathon races. In addition, disposable strip has the advantage of easy manufacture and low cost, making their use an inexpensive analysis compared with the traditional method. Finally, the performance of complex instruments applied in traditional CLIA methods depends on skilled and properly trained technicians, while the ease of performing the assessment with the present disposable strip-based method makes it a suitable system for POCT that does not require prior training.

The present analysis only took factors associated with technical success into account, without considering any risk factors, including hypertension, smoking and family history of coronary artery disease (26). It is necessary to validate the results with the inclusion of these risk factors in further investigations. Although the present study only included limited factors, it had similar accuracy in the diagnosis of IHD to that of the traditional CLIA method.

\section{Acknowledgements}

The authors thank Dr Chen Xinglin (Department of Epidemiology and Biostatistics, $\mathrm{X} \& \mathrm{Y}$ solutions Inc. in Boston) for guiding the use of EmpowerStats software.

\section{Funding}

This project was supported by the National Natural Science Foundation of China (grant no. 21602138), the Natural Science Foundation of Guangdong (grant nos. 2016A030313029, 2016A030310032 and 2017A030313668), Sanming Project of Medicine in Shenzhen (grant nos. SZSM201612031 and SZXJ2017076), Health and Family Planning Commission of Shenzhen Municipality (grant no. 201605009) and the Shenzhen Municipal Government of China (grant nos. GJHZ20160301163138685, JCYJ20170817171808368 and JCYJ20170818085657917).

\section{Availability of data and materials}

The data used and/or analyzed during the present study are available from the corresponding author on reasonable request.

\section{Authors' contributions}

SH and NX conceived and designed the study. LQ made the draft and $\mathrm{SH}$ revised the manuscript. SH, LQ and DZ performed the experiments. $\mathrm{SH}$ and $\mathrm{XH}$ analysed the data. All authors read and approved the manuscript.

\section{Ethics approval and consent to participate}

The study was performed in accordance with the Declaration of Helsinki and was approved by the Ethics Committee of Shenzhen Second People's Hospital (Shenzhen, China). During data collection, all patients were informed to fully understand the study and signed the consent documents referenced from the Chinese Clinical Trial registry (http://www.chictr.org. $\mathrm{cn} /$, no. ChiCTR2000032549). All of the experiments were performed in accordance with relevant guidelines and regulations.

\section{Patient consent for publication}

Not applicable.

\section{Competing interests}

The authors declare that they have no competing interests.

\section{References}

1. Joseph P, Leong D, McKee M, Anand SS, Schwalm JD, Teo K, Mente A and Yusuf S: Reducing the global burden of cardiovascular disease, part 1: The epidemiology and risk factors. Circ Res 121: 677-694, 2017 
2. Lamelas PM, Yusuf S and Schwalm JD: Effective approaches to address the global cardiovascular disease burden. Curr Opin Cardiol 32: 557-566, 2017.

3. Mozaffarian D, Benjamin EJ, Go AS, Arnett DK, Blaha MJ, Cushman M, Das SR, de Ferranti S, Despres JP, Fullerton HJ, et al: Executive summary: Heart disease and stroke statistics-2016 update: A report from the American heart association. Circulation 133: 447-454, 2016.

4. Roth GA, Johnson C, Abajobir A, Abd-Allah F, Abera SF, Abyu G, Ahmed M, Aksut B, Alam T, Alam K, et al: Global, regional, and national burden of cardiovascular diseases for 10 causes, 1990 to 2015. J Am Coll Cardiol 70: 1-25, 2017.

5. World Health Organization (WHO): Global health estimates: Deaths by cause, age, sex and country, 2000-2012. WHO, Geneva, 2014.

6. Lippi G, Franchini M and Cervellin G: Diagnosis and management of ischemic heart disease. Semin Thromb Hemost 39: 202-213, 2013.

7. Safdar B, Nagurney JT, Anise A, DeVon HA, D'Onofrio G, Hess EP, Hollander JE, Legato MJ, McGregor AJ, Scott J, et al: Gender-specific research for emergency diagnosis and management of ischemic heart disease: Proceedings from the 2014 academic emergency medicine consensus conference cardiovascular research workgroup. Acad Emerg Med 21: 1350-1360, 2014.

8. de Winter RJ, Koster RW, Sturk A and Sanders GT: Value of myoglobin, troponin T, and CK-MBmass in ruling out an acute myocardial infarction in the emergency room. Circulation 92: 3401-3407, 1995.

9. Scebba F, Papale M, Rocchiccioli S, Ucciferri N, Bigazzi F, Sampietro T, Carpeggiani C, L'Abbate A, Coceani F and Angeloni D: Differential proteome profile in ischemic heart disease: Prognostic value in chronic angina versus myocardial infarction. A proof of concept. Clin Chim Acta 471: 68-75, 2017.

10. Hachey BJ, Kontos MC, Newby LK, Christenson RH, Peacock WF, Brewer KC and Mccord J: Trends in use of biomarker protocols for the evaluation of possible myocardial infarction. J Am Heart Assoc 6: e005852, 2017.

11. Haeck JD, Verouden NJ, Kuijt WJ, Koch KT, Van Straalen JP, Fischer J, Groenink M, Bilodeau L, Tijssen JG, Krucoff MW and De Winter RJ: Comparison of usefulness of $\mathrm{N}$-terminal pro-brain natriuretic peptide as an independent predictor of cardiac function among admission cardiac serum biomarkers in patients with anterior wall versus nonanterior wall ST-segment elevation myocardial infarction. Am J Cardiol 105: 1065-1069, 2010.

12. Hetland $\mathrm{O}$ and Dickstein $\mathrm{K}$ : Cardiac markers in the early hours of acute myocardial infarction: Clinical performance of creatine kinase, creatine kinase MB isoenzyme (activity and mass concentration), creatine kinase MM and MB subform ratios, myoglobin and cardiac troponin T. Scand J Clin Lab Invest 56: 701-713, 1996.

13. Jolly SS, Shenkman H, Brieger D, Fox KA, Yan AT, Eagle KA, Steg PG, Lim KD, Quill A and Goodman SG; GRACE Investigators: Quantitative troponin and death, cardiogenic shock, cardiac arrest and new heart failure in patients with non-ST-segment elevation acute coronary syndromes (NSTE ACS): Insights from the global registry of acute coronary events. Heart 97: 197-202, 2011.

14. Zeller T, Ojeda F, Brunner FJ, Peitsmeyer P, Munzel T, Binder H, Pfeiffer N, Michal M, Wild PS, Blankenberg S and Lackner KJ: High-sensitivity cardiac troponin I in the general population-defining reference populations for the determination of the 99th percentile in the gutenberg health study. Clin Chem Lab Med 53: 699-706, 2015.
15. Penttila I, Penttila K and Rantanen T: Laboratory diagnosis of patients with acute chest pain. Clin Chem Lab Med 38: 187-197, 2000.

16. Weigl B, Domingo G, Labarre P and Gerlach J: Towards nonand minimally instrumented, microfluidics-based diagnostic devices. Lab Chip 8: 1999-2014, 2008.

17. Chen A and Yang S: Replacing antibodies with aptamers in lateral flow immunoassay. Biosens Bioelectron 71: 230-242, 2015.

18. Chen J, Zhou S and Wen J: Disposable strip biosensor for visual detection of $\mathrm{Hg}(2+)$ based on $\mathrm{Hg}(2+)$-triggered toehold binding and exonuclease III-assisted signal amplification. Anal Chem 86: 3108-3114, 2014.

19. Yu L, Shi Z, Fang C, Zhang Y, Liu Y and Li C: Disposable lateral flow-through strip for smartphone-camera to quantitatively detect alkaline phosphatase activity in milk. Biosens Bioelectron 69: 307-315, 2015.

20. Weng CW, Hsieh BC, Hou YT and Cheng TJ: Determination of hematocrit by voltage-induced hemolysis on a disposable electrochemical sensing strip. Analyst 140: 6619-6624, 2015.

21. Li X, Wang X, Fang T, Zhang L and Gong J: Disposable photoelectrochemical sensing strip for highly sensitive determination of perfluorooctane sulfonyl fluoride on functionalized screen-printed carbon electrode. Talanta 181: 147-153, 2018.

22. Braunwald E, Antman EM, Beasley JW, Califf RM, Cheitlin MD, Hochman JS, Jones RH, Kereiakes D, Kupersmith J, Levin TN, et al: ACC/AHA 2002 guideline update for the management of patients with unstable angina and non-ST-segment elevation myocardial infarction-summary article: A report of the American college of cardiology/American heart association task force on practice guidelines (committee on the management of patients with unstable angina). J Am Coll Cardiol 40: 1366-1374, 2002.

23. Christenson RH and Azzazy HM: Cardiac point of care testing: A focused review of current national academy of clinical biochemistry guidelines and measurement platforms. Clin Biochem 42: 150-157, 2009.

24. Storrow AB, Apple FS, Wu AHB, Jesse RL, Francis GS, Christenson RH, Cannon CP, Morrow DA, Newby LK, Ravkilde $\mathrm{J}$, et al: National academy of clinical biochemistry laboratory medicine practice guidelines: Point of care testing, oversight, and administration of cardiac biomarkers for acute coronary syndromes. Point Care 6: 215-222, 2007.

25. McRae AD, Innes G, Graham M, Lang E, Andruchow JE, Yang H, Ji Y, Vatanpour S, Southern DA, Wang D, et al: Comparative evaluation of 2-hour rapid diagnostic algorithms for acute myocardial infarction using high-sensitivity cardiac troponin T. Can J Cardiol 33: 1006-1012, 2017.

26. Mancini GB, Gosselin G, Chow B, Kostuk W, Stone J, Yvorchuk KJ, Abramson BL, Cartier R, Huckell V, Tardif JC, et al: Canadian cardiovascular society guidelines for the diagnosis and management of stable ischemic heart disease. Can J Cardiol 30: 837-849, 2014.

This work is licensed under a Creative Commons Attribution-NonCommercial-NoDerivatives 4.0 International (CC BY-NC-ND 4.0) License. 